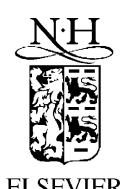

\title{
Quantum mechanical symmetries and topological invariants
}

\author{
K. Aghababaei Samani ${ }^{\mathrm{a}}$, A. Mostafazadeh ${ }^{\mathrm{b}, *}$ \\ a Institute for Advanced Studies in Basic Sciences, 45195-159 Gava Zang, Zanjan, Iran \\ b Department of Mathematics, Koç University, Rumelifeneri Yolu, 80910 Sariyer, Istanbul, Turkey
}

Received 3 July 2000; accepted 22 November 2000

\begin{abstract}
We give the definition and explore the algebraic structure of a class of quantum symmetries, called topological symmetries, which are generalizations of supersymmetry in the sense that they involve topological invariants similar to the Witten index. A topological symmetry (TS) is specified by an integer $n>1$, which determines its grading properties, and an $n$-tuple of positive integers $\left(m_{1}, m_{2}, \ldots, m_{n}\right)$. We identify the algebras of supersymmetry, $p=2$ parasupersymmetry, and fractional supersymmetry of order $n$ with those of the $\mathbb{Z}_{2}$-graded TS of type $(1,1), \mathbb{Z}_{2}$-graded TS of type $(2,1)$, and $\mathbb{Z}_{n}$-graded TS of type $(1,1, \ldots, 1)$, respectively. We also comment on the mathematical interpretation of the topological invariants associated with the $\mathbb{Z}_{n}$-graded TS of type $(1,1, \ldots, 1)$. For $n=2$, the invariant is the Witten index which can be identified with the analytic index of a Fredholm operator. For $n>2$, there are $n$ independent integer-valued invariants. These can be related to differences of the dimension of the kernels of various products of $n$ operators satisfying certain conditions. @ 2001 Elsevier Science B.V. All rights reserved.
\end{abstract}

\section{Introduction}

Supersymmetric quantum mechanics was originally introduced as a toy model used to study some of the features of supersymmetric field theories [1]. This simple toy model has, however, proven to be a very useful tool in dealing with a variety of problems in quantum and statistical mechanics [2-4]. Supersymmetric quantum mechanics has also been used to derive some of the very basic results of differential topology. Among these are the supersymmetric derivation of the Morse inequalities [5] and supersymmetric proofs of the Atiyah-Singer index theorem [6].

\footnotetext{
* Corresponding author.

E-mail addresses: samani@iasbs.ac.ir (K. Aghababaei Samani), amostafazadeh@ku.edu.tr (A. Mostafazadeh).
} 
The relationship between supersymmetry and topological invariants such as the indices of the elliptic operators is our main motivation for seeking general quantum mechanical symmetries with topological properties similar to those of supersymmetry.

Various generalizations of supersymmetry have been considered in the literature [712]. Among these only extended and generalized supersymmetries [12] and a certain class of $p=2$ parasupersymmetries [13] are known to share the topological characteristics of supersymmetry.

The strategy pursued in this article is as follows. First, we introduce the notion of a topological symmetry (TS) by formulating a set of basic principles that ensure the desired topological properties. Then, we investigate the underlying algebraic structure of these symmetries. This is necessary for seeking a mathematical interpretation of the corresponding topological invariants.

We have recently reported our preliminary results on $\mathbb{Z}_{2}$-graded TSs of type $(1,1)$ and $(2,1)$ in [14]. The purpose of the present article is to generalize the results of [14] to arbitrary $\mathbb{Z}_{n}$-graded TSs.

The organization of the article is as follows. In Section 2, we give the definition of a general $\mathbb{Z}_{n}$-graded TS and introduce the associated topological invariants. In Section 3, we consider the case of $n=2$ and derive the algebra of a $\mathbb{Z}_{2}$-graded TS of arbitrary type $\left(m_{+}, m_{-}\right)$. In particular, we show that for $m_{-}=1$, the algebra can be reduced to the algebra of supersymmetry or $p=2$ parasupersymmetry. In Section 4 , we consider the $\mathbb{Z}_{n}$-graded TSs. Here we discuss the properties of the grading operator and derive the algebra of $\mathbb{Z}_{n}$-graded TSs of arbitrary type $\left(m_{1}, m_{2}, \ldots, m_{n}\right)$. In Section 5 , we comment on the mathematical interpretation of the topological invariants associated with TSs. In Section 6, we study some concrete examples of quantum systems possessing TSs. In Section 7, we summarize our results and present our concluding remarks. The appendix includes the proof of some of the mathematical results that we use in our analysis.

\section{2. $\mathbb{Z}_{n}$-graded TSs}

In order to describe the concept of a topological symmetry, we first give some basic definitions. In the following we shall only consider the quantum systems with a self-adjoint Hamiltonian $H$. We shall further assume that all the energy levels are at most finitely degenerate.

Definition 1. Let $n$ be an integer greater than $1, \mathcal{H}$ denote the Hilbert space of a quantum system, and $\mathcal{H}_{1}, \mathcal{H}_{2}, \ldots, \mathcal{H}_{n}$ be (nontrivial) subspaces of $\mathcal{H}$. Then a state vector is said to have definite $\operatorname{color} c_{\ell}$ iff it belongs to $\mathcal{H}_{\ell}$.

Definition 2. A quantum system is said to be $\mathbb{Z}_{n}$-graded iff its Hilbert space is the direct sum of $n$ of its (nontrivial) subspaces $\mathcal{H}_{\ell}$, and its Hamiltonian has a complete set of eigenvectors with definite color. 
Definition 3. Let $m_{\ell}$ be positive integers for all $\ell \in\{1,2, \ldots, n\}$, and $m:=\sum_{\ell=1}^{n} m_{\ell}$. Then a quantum system is said to possess a $\mathbb{Z}_{n}$-graded topological symmetry of type $\left(m_{1}, m_{2}, \ldots, m_{n}\right)$ iff the following conditions are satisfied.

(a) The quantum system is $\mathbb{Z}_{n}$-graded;

(b) The energy spectrum is nonnegative;

(c) For every positive energy eigenvalue $E$, there is a positive integer $\lambda_{E}$ such that $E$ is $\lambda_{E} m$-fold degenerate, and the corresponding eigenspaces are spanned by $\lambda_{E} m_{1}$ vectors of color $c_{1}, \lambda_{E} m_{2}$ vectors of color $c_{2}, \ldots$, and $\lambda_{E} m_{n}$ vectors of color $c_{n}$.

Definition 4. A topological symmetry is said to be uniform iff for all positive energy eigenvalues $E, \lambda_{E}=1$.

Theorem 1. Consider a quantum system possessing a $\mathbb{Z}_{n}$-graded topological symmetry of type $\left(m_{1}, m_{2}, \ldots, m_{n}\right)$, and let $n_{\ell}^{(0)}$ denote the number of zero-energy states of color $c_{\ell}$. Then for all $i, j \in\{1,2, \ldots, n\}$, the integers

$$
\Delta_{i j}:=m_{i} n_{j}^{(0)}-m_{j} n_{i}^{(0)}
$$

remain invariant under continuous symmetry-preserving changes of the quantum system. ${ }^{1}$

Proof. The proof of this theorem is essentially the same as the proof of the topological invariance of the Witten index of supersymmetry. A symmetry-preserving continuous change of the quantum system will preserve the particular degeneracy and grading structures of positive energy levels. Because under such a change an initial zero energy eigenstate can only become a positive energy eigenstate and vice versa, the only possible change in the number of zero energy eigenstates are the ones involving the changes of $n_{i}^{(0)}$ of the form

$$
n_{i}^{(0)} \rightarrow \tilde{n}_{i}^{(0)}:=n_{i}^{(0)}+k m_{i},
$$

where $k$ is an integer greater than or equal to $-n_{i}^{(0)} / m_{i}$. Moreover, such a change must occur simultaneously for all $n_{i}^{(0)}$, s, i.e., the transformation (2) is valid for all $i \in$ $\{1,2, \ldots, n\}$. Therefore, under such a symmetry-preserving change of the system,

$$
\begin{aligned}
\Delta_{i j} \rightarrow \tilde{\Delta}_{i j} & :=m_{i} \tilde{n}_{j}^{(0)}-m_{j} \tilde{n}_{i}^{(0)}=m_{i}\left(n_{j}^{(0)}+k m_{j}\right)-m_{j}\left(n_{i}^{(0)}+k m_{i}\right) \\
& =m_{i} n_{j}^{(0)}-m_{j} n_{i}^{(0)}=\Delta_{i j},
\end{aligned}
$$

i.e., $\Delta_{i j}$ 's remain invariant.

A direct consequence of Theorem 1 is that (the value of) any function of $\Delta_{i j}$ 's is a topological invariant of the system. In particular, $\Delta_{i j}$ 's are the basic topological invariants. A typical example of a derived invariant is

\footnotetext{
${ }^{1}$ Here we consider quantum systems whose energy spectrum depends on a set of continuous parameters. These parameters may be identified with coupling constants or geometric quantities entering the definition of the Hamiltonian and/or the Hilbert space. A continuous change of the system corresponds to a continuous change of these parameters.
} 


$$
\Delta:=\frac{1}{2} \sum_{i, j=1}^{n}\left(\Delta_{i j}\right)^{2} .
$$

Note that $\Delta$ is a measure of the existence of the zero-energy states.

Let us next observe that the topological symmetries are simple generalizations of supersymmetry. First we recall that the Hilbert space of a supersymmetric system is $\mathbb{Z}_{2}$-graded. We can relate the $\mathbb{Z}_{2}$-grading of the Hilbert space $\mathcal{H}$ to the existence of a "parity" or "grading" operator $\tau: \mathcal{H} \rightarrow \mathcal{H}$ satisfying

$$
\begin{aligned}
& \tau^{2}=1, \\
& \tau^{\dagger}=\tau, \\
& {[H, \tau]=0 .}
\end{aligned}
$$

Here we use a $\uparrow$ to denote the adjoint of the corresponding operator. We can identify the subspaces $\mathcal{H}_{1}$ and $\mathcal{H}_{2}$ with the eigenspaces of $\tau$,

$$
\mathcal{H}_{1}=\mathcal{H}_{+}, \quad \mathcal{H}_{2}=\mathcal{H}_{-}, \quad \mathcal{H}_{ \pm}:=\{\psi \in \mathcal{H} \mid \tau \psi= \pm \psi\} .
$$

Now, consider the superalgebra

$$
\begin{aligned}
& {[H, \mathcal{Q}]=0,} \\
& \frac{1}{2}\left\{\mathcal{Q}, \mathcal{Q}^{\dagger}\right\}=H, \\
& \mathcal{Q}^{2}=0,
\end{aligned}
$$

of supersymmetric quantum mechanics with one nonself-adjoint symmetry generator $\mathcal{Q}$ satisfying

$$
\{\mathcal{Q}, \tau\}=0 .
$$

It is well-known that using the superalgebra (8)-(10) together with the properties of the grading operator (4)-(6) and the symmetry generator (11), one can show that the conditions (a)-(c) of Definition 3, with $n=2$ and $m_{1}=m_{2}=1$, are satisfied. Therefore, supersymmetry is a $\mathbb{Z}_{2}$-graded TS of type $(1,1)$. For a $\mathbb{Z}_{2}$-graded TS of type $(1,1)$, there is a single basic topological invariant, namely, $\Delta_{11}$. This is precisely the Witten index.

\section{Algebraic structure of $\mathbb{Z}_{2}$-graded TSs}

In this section we shall explore the algebraic structure of the $\mathbb{Z}_{2}$-graded TSs that fulfil the following conditions.

- The $\mathbb{Z}_{2}$-grading is achieved by a grading operator $\tau$ satisfying (4)-(6);

- There is a single nonself-adjoint symmetry generator $\mathcal{Q}$;

$-\mathcal{Q}$ is an odd operator, i.e., it satisfies Eq. (11).

We shall only treat the case of the uniform TSs. The algebraic structure of nonuniform TSs is easily obtained from that of the uniform topological symmetries (UTSs). In fact, the algebraic relations defining uniform and nonuniform TSs of the same type are identical. 
In order to obtain the algebraic structures that support TSs, we shall use the information on the degeneracy structure of the corresponding systems and the properties of the grading operator and the symmetry generator to construct matrix representations of the relevant operators in the energy eigenspaces $\mathcal{H}_{E}$ with positive eigenvalue $E$. We shall use the notation $O^{E}$ for the restriction of an operator $O$ onto the eigenspace $\mathcal{H}_{E}$. Throughout this article $E$ stands for a positive energy eigenvalue. The zero-energy eigenspace (kernel of $H$ ) is denoted by $\mathcal{H}_{0}$.

In view of Eqs. (6) and (8), $\tau^{E}$ and $\mathcal{Q}^{E}$ are $m \times m$ matrices acting in $\mathcal{H}_{E}$. We also have the trivial identity: $H^{E}=E I_{m}$, where $I_{m}$ denotes the $m \times m$ unit matrix.

Next, we introduce the self-adjoint symmetry generators

$$
Q_{1}:=\frac{1}{\sqrt{2}}\left(\mathcal{Q}+\mathcal{Q}^{\dagger}\right) \quad \text { and } \quad Q_{2}:=\frac{-i}{\sqrt{2}}\left(\mathcal{Q}-\mathcal{Q}^{\dagger}\right),
$$

where $i:=\sqrt{-1}$. Note that because $\tau$ is self-adjoint, we have

$$
\left\{Q_{j}, \tau\right\}=0 \text { for } j \in\{1,2\} .
$$

Now in view of Eq. (6), we can choose a basis in $\mathcal{H}_{E}$ in which $\tau$ is diagonal. Then using Eqs. (4), (12), (13), and the self-adjointness of $Q_{j}$, we obtain the following matrix representations for $\tau^{E}, Q_{j}^{E}$, and $\mathcal{Q}^{E}$.

$$
\begin{aligned}
\tau^{E} & =\operatorname{diag}(\underbrace{1,1, \ldots, 1}_{m_{+} \text {times }}, \underbrace{-1,-1, \ldots,-1}_{m_{\text {-times }}}), \\
Q_{j}^{E} & =\left(\begin{array}{cc}
0 & A_{j} \\
A_{j}^{\dagger} & 0
\end{array}\right), \\
\mathcal{Q}^{E} & =\frac{1}{\sqrt{2}}\left(\begin{array}{cc}
0 & A_{1}+i A_{2} \\
A_{1}^{\dagger}+i A_{2}^{\dagger} & 0
\end{array}\right),
\end{aligned}
$$

where " $\operatorname{diag}(\cdots)$ " stands for a diagonal matrix with diagonal entries “...", 0's denote appropriate zero matrices, and $A_{j}$ are $m_{+} \times m_{-}$complex matrices.

The next step is to find general identities satisfied by $Q_{j}^{E}$ and $\mathcal{Q}^{E}$ for all $E>0$. In order to derive the simplest such identities we appeal to the Cayley-Hamilton theorem of linear algebra. This theorem states that an $m \times m$ matrix $Q$ satisfies its characteristic equations, $\mathcal{P}_{Q}(Q)=0$, where $\mathcal{P}_{Q}(x):=\operatorname{det}\left(x I_{m}-Q\right)$ is the characteristic polynomial for $Q$. Using this theorem we can prove the following lemma. The proof is given in the appendix.

Lemma 1. Let $m_{ \pm}$be positive integers, $m:=m_{+}+m_{-}$, and $Q$ be an $m \times m$ matrix of the form:

$$
Q=\left(\begin{array}{ll}
0 & X \\
Y & 0
\end{array}\right),
$$

where $X$ and $Y$ are $m_{+} \times m_{-}$and $m_{-} \times m_{+}$complex matrices. Let $\mathcal{P}_{X Y}(x)$ and $\mathcal{P}_{Y X}(x)$ denote the characteristic polynomials for $X Y$ and $Y X$, respectively. Then $\mathcal{P}_{Y X}\left(Q^{2}\right) Q=$ $\mathcal{P}_{X Y}\left(Q^{2}\right) Q=0$. Furthermore, if $m_{+}=m_{-}$, then $\mathcal{P}_{Y X}\left(Q^{2}\right)=\mathcal{P}_{X Y}\left(Q^{2}\right)=0$. 
Applying this lemma to $Q_{j}^{E}$ and $\mathcal{Q}^{E}$, we find for $m_{+}=m_{-}$

$$
\begin{gathered}
\mathcal{P}_{j}\left[\left(Q_{j}^{E}\right)^{2}\right]=0, \\
\mathcal{P}\left[\left(\mathcal{Q}^{E}\right)^{2}\right]=0,
\end{gathered}
$$

and for $m_{+}>m_{-}$

$$
\begin{gathered}
\mathcal{P}_{j}\left[\left(Q_{j}^{E}\right)^{2}\right] Q_{j}^{E}=0, \\
\mathcal{P}\left[\left(\mathcal{Q}^{E}\right)^{2}\right] \mathcal{Q}^{E}=0,
\end{gathered}
$$

where $\mathcal{P}_{j}(x)$ and $\mathcal{P}(x)$ denote the characteristic polynomials of $A_{j}^{\dagger} A_{j}$ and $\left(A_{1}^{\dagger}+\right.$ $\left.i A_{2}^{\dagger}\right)\left(A_{1}+i A_{2}\right) / 2$, respectively.

For $m_{-}>m_{+}$, the roles of $A_{j}$ and $A_{j}^{\dagger}$ are interchanged. We shall, therefore, restrict our attention to the case where $m_{+} \geqslant m_{-}$.

We can write Eqs. (18)-(21) in terms of the roots of the characteristic polynomials $\mathcal{P}_{j}(x)$ and $\mathcal{P}(x)$. This yields

$$
\begin{aligned}
& {\left[\left(Q_{j}^{E}\right)^{2}-\mu_{j 1}^{E} I_{m}\right]\left[\left(Q_{j}^{E}\right)^{2}-\mu_{j 2}^{E} I_{m}\right] \cdots\left[\left(Q_{j}^{E}\right)^{2}-\mu_{j m_{-}}^{E} I_{m}\right]\left(Q_{j}^{E}\right)^{1-\delta\left(m_{+}, m_{-}\right)}=0,} \\
& {\left[\left(\mathcal{Q}^{E}\right)^{2}-\kappa_{1}^{E} I_{m}\right]\left[\left(\mathcal{Q}^{E}\right)^{2}-\kappa_{2}^{E} I_{m}\right] \cdots\left[\left(\mathcal{Q}^{E}\right)^{2}-\kappa_{m_{-}}^{E} I_{m}\right]\left(\mathcal{Q}^{E}\right)^{1-\delta\left(m_{+}, m_{-}\right)}=0,}
\end{aligned}
$$

where $\mu_{j \ell}^{E}$ and $\kappa_{\ell}^{E}$ are the roots ${ }^{2}$ of $\mathcal{P}_{j}(x)$ and $\mathcal{P}(x)$, respectively, and

$$
\delta\left(m_{+}, m_{-}\right)=\delta_{m_{+}, m_{-}}:= \begin{cases}1 & \text { for } m_{+}=m_{-}, \\ 0 & \text { for } m_{+} \neq m_{-} .\end{cases}
$$

In order to promote Eqs. (22) and (23) to operator relations, we introduce the operators $M_{j \ell}$ and $\mathcal{K}_{\ell}$ (for each $j \in\{1,2\}$ and $\ell \in\left\{1,2, \ldots, m_{-}\right\}$) which commute with $H$ and have the representations:

$$
M_{j \ell}^{E}=\mu_{j \ell}^{E} I_{m} \quad \text { and } \quad \mathcal{K}_{\ell}^{E}=\kappa_{\ell}^{E} I_{m},
$$

in $\mathcal{H}_{E}$. We then deduce from Eqs. (22), (23) and (24) that $M_{j \ell}$ and $\mathcal{K}_{\ell}$ must commute with $\tau$ and $Q_{j}$. Furthermore, they should satisfy the algebra

$$
\begin{aligned}
& \left(Q_{j}^{2}-M_{j 1}\right)\left(Q_{j}^{2}-M_{j 2}\right) \cdots\left(Q_{j}^{2}-M_{j m_{-}}\right) Q_{j}^{1-\delta\left(m_{+}, m_{-}\right)}=0, \\
& \left(\mathcal{Q}^{2}-\mathcal{K}_{1}\right)\left(\mathcal{Q}^{2}-\mathcal{K}_{2}\right) \cdots\left(\mathcal{Q}^{2}-\mathcal{K}_{m_{-}}\right) \mathcal{Q}^{1-\delta\left(m_{+}, m_{-}\right)}=0 .
\end{aligned}
$$

Note that the roots $\kappa_{\ell}^{E}$ and $\mu_{j \ell}^{E}$ are defined using the matrices $A_{j}$. This suggests that the operators $M_{j \ell}$ and $\mathcal{K}_{\ell}$ are not generally independent. Furthermore, because $A_{j}^{\dagger} A_{j}$ are Hermitian matrices, the roots $\mu_{j \ell}^{E}$, which are in fact the eigenvalues of $A_{j}^{\dagger} A_{j}$, are real. This in turn suggests that the operators $M_{j \ell}$ are self-adjoint.

In summary, the algebra of general $\mathbb{Z}_{2}$-graded topological symmetry of type $\left(m_{+}, m_{-}\right)$ which is generated by one odd nonself-adjoint generator $\mathcal{Q}$ is given by Eqs. (25) and (26) where $m_{+}$is assumed (without loss of generality) not to be smaller than $m_{-}$, the operators

\footnotetext{
${ }^{2}$ Note that the roots are not necessarily distinct.
} 
$M_{j \ell}$ and $\mathcal{K}_{\ell}$ commute with $H, \tau$ and $\mathcal{Q}$, and $M_{j \ell}$ are self-adjoint. Moreover, $M_{j \ell}$ and $\mathcal{K}_{\ell}$ have similar degeneracy structure as the Hamiltonian ${ }^{3}$ (at least for positive energy eigenvalues). In particular, it might be possible to express $H$ as a function of $M_{j \ell}$ and $\mathcal{K}_{\ell}$.

In order to elucidate the role of the operators $M_{j \ell}$ and $\mathcal{K}_{\ell}$ and their relation to the Hamiltonian, we shall next consider the $\mathbb{Z}_{2}$-graded UTSs of type $\left(m_{+}, 1\right)$.

If $m_{-}=1$, then $A_{j}^{\dagger} A_{j}$ and $\left(A_{1}^{\dagger}+i A_{2}^{\dagger}\right)\left(A_{1}+i A_{2}\right) / 2$ are, respectively, real and complex scalars. In this case, $\mathcal{P}_{j}(x)=x-\mu_{j}^{E}$ and $\mathcal{P}(x)=x-\kappa^{E}$, where

$$
\begin{aligned}
\mu_{j}^{E} & =A_{j}^{\dagger} A_{j}, \\
\kappa^{E} & =\frac{1}{2}\left(A_{1}^{\dagger}+i A_{2}^{\dagger}\right)\left(A_{1}+i A_{2}\right)=\frac{1}{2}\left[\left(A_{1}^{\dagger} A_{1}-A_{2}^{\dagger} A_{2}\right)+i\left(A_{1}^{\dagger} A_{2}+A_{2}^{\dagger} A_{1}\right)\right],
\end{aligned}
$$

and the algebra (25) and (26) takes the form

$$
\begin{aligned}
& \left(Q_{j}^{2}-M_{j}\right) Q_{j}^{1-\delta\left(m_{+}, 1\right)}=0, \\
& \left(\mathcal{Q}^{2}-\mathcal{K}\right) \mathcal{Q}^{1-\delta\left(m_{+}, 1\right)}=0 .
\end{aligned}
$$

Here we have used the abbreviated notation: $M_{j}=M_{j 1}$ and $\mathcal{K}=\mathcal{K}_{1}$.

Next, we define the self-adjoint operators

$$
K_{1}=\mathcal{K}+\mathcal{K}^{\dagger} \quad \text { and } \quad K_{2}=-i\left(\mathcal{K}-\mathcal{K}^{\dagger}\right) .
$$

In view of Eqs. (24) and (27) we have

$$
M_{2}=M_{1}-K_{1} \text {. }
$$

In the following we shall consider the cases $m_{+}=1$ and $m_{+}>1$ separately.

\section{1. $\mathbb{Z}_{2}$-graded UTS of type $(1,1)$}

Setting $m_{+}=1$ in Eqs. (28) and (29), we find

$$
\begin{aligned}
Q_{j}^{2} & =M_{j}, \\
\mathcal{Q}^{2} & =\mathcal{K} .
\end{aligned}
$$

If we express $\mathcal{Q}$ in terms of $Q_{j}$ and use Eqs. (30) and (31), we can write Eqs. (32) and (33) in the form

$$
\begin{aligned}
& Q_{1}^{2}=M_{1}, \\
& Q_{2}^{2}=M_{1}-K_{1}, \\
& \left\{Q_{1}, Q_{2}\right\}=K_{2} .
\end{aligned}
$$

Now, we observe that Eqs. (34)-(36) remain form-invariant under the linear transformations of the form

\footnotetext{
${ }^{3}$ The degeneracy structure of these operators will be the same as that of the Hamiltonian, if their eigenvalues $\mu_{j \ell}^{E}$ and $\kappa_{\ell}^{E}$ are distinct for different $E$.
} 


$$
\begin{aligned}
& Q_{1} \rightarrow \widetilde{Q}_{1}=a Q_{1}+b Q_{2}, \\
& Q_{2} \rightarrow \widetilde{Q}_{2}=c Q_{1}+d Q_{2},
\end{aligned}
$$

where $a, b, c$ and $d$ are self-adjoint operators commuting with all other operators. More specifically, $\widetilde{Q}_{j}$ satisfy Eqs. (34)-(36) provided that $M_{1}$ and $K_{j}$ are transformed according to

$$
\begin{aligned}
M_{1} \rightarrow \widetilde{M}_{1} & :=\left(a^{2}+b^{2}\right) M_{1}-b^{2} K_{1}+a b K_{2}, \\
K_{1} \rightarrow \widetilde{K}_{1} & :=\left(a^{2}+b^{2}-c^{2}-d^{2}\right) M_{1}+\left(d^{2}-b^{2}\right) K_{1}+(a b-c d) K_{2}, \\
K_{2} \rightarrow \widetilde{K}_{2} & :=2(a c+b d) M_{1}-2 b d K_{1}+(a d+b c) K_{2} .
\end{aligned}
$$

In particular, there are transformations of the form (38) for which $\widetilde{K}_{j}=0$. These correspond to the choices for $a, b, c$ and $d$ that satisfy (either of)

$$
\frac{a+i c}{b+i d}=-\frac{K_{2}}{2 M_{1}} \pm i \sqrt{1-\frac{K_{1}}{M_{1}}-\frac{K_{2}^{2}}{4 M_{1}^{2}}} .
$$

One can use the representations of $K_{j}$ and $M_{1}$ in the eigenspaces $\mathcal{H}_{E}$ to show that the terms in the square root in (41) yield a positive self-adjoint operator, provided that the kernel of $M_{1}$ is a subspace of the zero-energy eigenspace $\mathcal{H}_{0}$.

The above analysis shows that we can reduce the general algebra (34)-(36) to the special case where $K_{j}=0$. Writing this algebra in terms of $\mathcal{Q}$, we obtain the superalgebra (8)(10) with $M_{1}$ replacing $H$. In other words, if we identify the Hamiltonian with $M_{1}$, which we can always do, the algebra of $\mathbb{Z}_{2}$-graded topological symmetry of type $(1,1)$ reduces to that of supersymmetry.

\section{2. $\mathbb{Z}_{2}$-graded UTSs of type $\left(m_{+}, 1\right)$ with $m+>1$}

If $M_{+}>1$, then Eqs. (28) and (29) take the form

$$
\begin{aligned}
Q_{j}^{3} & =M_{j} Q_{j}, \\
\mathcal{Q}^{3} & =\mathcal{K} \mathcal{Q} .
\end{aligned}
$$

Again we express $\mathcal{Q}$ in terms of $Q_{j}$ and use Eqs. (30) and (31) to write (42) and (43) in the form

$$
\begin{aligned}
& Q_{1}^{3}=M_{1} Q_{1}, \\
& Q_{2}^{3}=\left(M_{1}-K_{1}\right) Q_{2}, \\
& Q_{2} Q_{1} Q_{2}+\left\{Q_{1}, Q_{2}^{2}\right\}=\left(M_{1}-K_{1}\right) Q_{1}+K_{2} Q_{2}, \\
& Q_{1} Q_{2} Q_{1}+\left\{Q_{2}, Q_{1}^{2}\right\}=M_{1} Q_{2}+K_{2} Q_{1} .
\end{aligned}
$$

It is remarkable that these relations are also invariant under the transformations (37) and (38)-(40). Therefore, again we can reduce our analysis to the special case where $K_{j}=0$. Substituting zero for $K_{j}$ in Eqs. (44)-(47), and writing them in terms of $\mathcal{Q}$, we obtain 


$$
\begin{aligned}
& {\left[M_{1}, \mathcal{Q}\right]=0,} \\
& \left\{\mathcal{Q}^{2} Q^{\dagger}\right\}+\mathcal{Q} \mathcal{Q}^{\dagger} \mathcal{Q}=2 M_{1} \mathcal{Q}, \\
& \mathcal{Q}^{3}=0 .
\end{aligned}
$$

This is precisely the algebra of $p=2$ parasupersymmetry of Rubakov and Spiridonov [7] with $H$ replaced by $M_{1} / 2$. Hence, if we identify $H$ with $M_{1} / 2$, which we can always do, the algebra of $\mathbb{Z}_{2}$-graded topological symmetry of type $\left(m_{+}, 1\right)$ with $m_{+}>1$ reduces to that of the $p=2$ parasupersymmetry.

As shown in Ref. [15], one can use the algebra (48)-(50) of $p=2$ parasupersymmetry and properties of the grading operator (4)-(6) and (13) to obtain the general degeneracy structure of a $p=2$ parasupersymmetric system. In general the algebra of $p=2$ parasupersymmetry does not imply the particular degeneracy structure of the $\mathbb{Z}_{2}$-graded UTS of type $\left(m_{+}, 1\right)$, even for $m_{+}=2$. Therefore, the $\mathbb{Z}_{2}$-graded UTS of type $(2,1)$ is a subclass of the general $p=2$ parasupersymmetries. As argued in Refs. [15] and [13], these are parasupersymmetries for which an analog of the Witten index can be defined.

In Ref. [15] it is also shown that the positive energy eigenvalues of a $p=2$ parasupersymmetric system can at most be triply degenerate, provided that the eigenvalues of $Q_{1}^{E}$ for all $E>0$ are nondegenerate. This means that the $\mathbb{Z}_{2}$-graded TSs of type $\left(m_{+}, 1\right)$ with $m_{+}>2$ occur only if $Q_{1}^{E}$ have degenerate eigenvalues for all $E>0$. This suggests the presence of further (even) symmetry generators $L_{a}$ which would commute with $Q_{1}$ and label the basis eigenvectors within the degeneracy subspaces of $Q_{1}$. The existence of these generators is an indication that the $\mathbb{Z}_{2}$-graded TSs of type $\left(m_{+}, 1\right)$ with $m_{+}>2$ are not uniform.

\subsection{Special $\mathbb{Z}_{2}$-graded TSs}

The analysis of the $\mathbb{Z}_{2}$-graded TSs of type $\left(m_{+}, 1\right)$ shows that the corresponding algebras can be reduced to a simplified special case by a redefinition of the symmetry generators $Q_{j}$. This raises the question whether this is also possible for the general $\mathbb{Z}_{2}$-graded TSs of type $\left(m_{+}, m_{-}\right)$. The reduction made in the case of $\mathbb{Z}_{2}$-graded TSs of type $\left(m_{+}, 1\right)$ has its roots in the form of the matrix representation of the corresponding symmetry generators in $\mathcal{H}_{E}$. For a $\mathbb{Z}_{2}$-graded UTS of arbitrary type $\left(m_{+}, m_{-}\right)$, a similar reduction, which eliminates the operators $\mathcal{K}_{\ell}$ in Eq. (26), is possible, if we can find a transformation $Q_{j} \rightarrow \widetilde{Q}_{j}$ which satisfies the following conditions.

(1) The transformed generators $\widetilde{Q}_{j}$ have the representation

in $\mathcal{H}_{E}$.

$$
\widetilde{Q}_{j}^{E}=\left(\begin{array}{cc}
0 & \tilde{A}_{j} \\
\tilde{A}_{j}^{\dagger} & 0
\end{array}\right),
$$

(2) For all energy eigenvalues $E>0$, the corresponding matrices $\tilde{A}_{j}$ which define $\widetilde{Q}_{j}^{E}$ satisfy $\tilde{A}_{2}=U \tilde{A}_{1}$, where $U$ is an $m_{+} \times m_{+}$unitary and anti-Hermitian matrix.

The first condition is necessary for the invariance of the algebra (25)-(26). It is satisfied by the linear transformations: $Q_{j}^{E} \rightarrow \widetilde{Q}_{j}^{E}=T_{j} Q_{j}^{E} T_{j}^{\dagger}$ where $T_{j}$ are $m \times m$ matrices of the form 


$$
T_{j}=\left(\begin{array}{cc}
T_{j+} & 0 \\
0 & T_{j-}
\end{array}\right),
$$

and $T_{j \pm}$ are $m_{ \pm} \times m_{ \pm}$matrices. Under such a transformation $A_{j}$ transform according to $A_{j} \rightarrow \tilde{A}_{j}:=T_{j+} A_{j} T_{j-}^{\dagger}$.

The second condition implies that the transformed matrices $\tilde{A}_{j}$ satisfy

$$
\begin{aligned}
\left(\tilde{A}_{1}^{\dagger}+i \tilde{A}_{2}^{\dagger}\right)\left(\tilde{A}_{1}+i \tilde{A}_{2}\right) & =A_{1}^{\dagger}\left(I_{m_{+}}+i U^{\dagger}\right)\left(I_{m_{+}}+i U\right) A_{1} \\
& =A_{1}^{\dagger}\left[\left(I_{m_{+}}-U^{\dagger} U\right)+i\left(U+U^{\dagger}\right)\right] A_{1}=0,
\end{aligned}
$$

where we have used the unitarity and anti-Hermiticity of $U$. The latter relation indicates that for the transformed system $\kappa_{\ell}^{E}=0$ and $\mu_{2 \ell}^{E}=\mu_{1 \ell}^{E}$, for all $E$ and $\ell$. Hence $\mathcal{K}_{\ell}$ can be identified with the zero operator and $M_{2 \ell}=M_{1 \ell}=: M_{\ell}$. Furthermore, one can check that (51) implies $\left(\widetilde{\mathcal{Q}}^{E}\right)^{3-\delta\left(m_{+}, m_{-}\right)}=0$. Therefore, the transformed generators satisfy

$$
\begin{aligned}
& \left(\widetilde{Q}_{j}^{2}-M_{1}\right)\left(\widetilde{Q}_{j}^{2}-M_{2}\right) \cdots\left(\widetilde{Q}_{j}^{2}-M_{m_{-}}\right) \widetilde{Q}_{j}^{1-\delta\left(m_{+}, m_{-}\right)}=0, \\
& \widetilde{\mathcal{Q}}^{3-\delta\left(m_{+}, m_{-}\right)}=0 .
\end{aligned}
$$

We shall term such $\mathbb{Z}_{2}$-graded TSs, the special $\mathbb{Z}_{2}$-graded TSs.

\section{4. $\mathbb{Z}_{2}$-graded TSs with one self-adjoint generator}

The above analysis of $\mathbb{Z}_{2}$-graded TSs can be easily applied to the case where there is a single self-adjoint generator $Q$. In fact, one can read off the corresponding algebra from Eqs. (25) and (26). The result is

$$
\left(Q^{2}-M_{1}\right)\left(Q^{2}-M_{2}\right) \cdots\left(Q^{2}-M_{m_{-}}\right) Q^{1-\delta\left(m_{+}, m_{-}\right)}=0,
$$

where $M_{\ell}$ are self-adjoint operators commuting with $H, Q$ and $\tau$. For $m_{ \pm}=1$, this equation reduces to that of the $N=1 / 2$ supersymmetry, provided that we identify $M_{1}$ with the Hamiltonian $H$.

\section{Algebraic structure of $\mathbb{Z}_{n}$-graded TSs}

In the preceding section, we have used Definition 3 and the properties (4)-(6) and (11) of the $\mathbb{Z}_{2}$-grading operator $\tau$ to obtain the algebra of the $\mathbb{Z}_{2}$-graded TSs with one generator. The main guideline for postulating these properties were Definition 2 and the known grading structure of supersymmetric systems.

Similarly, in order to obtain the algebraic structure of the $\mathbb{Z}_{n}$-graded TSs, with $n>2$, we must first postulate the existence of an appropriate $\mathbb{Z}_{n}$-grading operator. In view of Definitions 1, 2 and 3, such a grading operator $\tau$ must commute with the Hamiltonian Eq. (6) holds - and have $n$-distinct eigenvalues $c_{\ell}$ with eigenspaces $\mathcal{H}_{\ell}$ satisfying $\mathcal{H}=$ $\mathcal{H}_{1} \oplus \mathcal{H}_{2} \oplus \cdots \oplus \mathcal{H}_{n}$. The simplest choice for $c_{\ell}$ are the $n$th roots of unity, e.g., $c_{\ell}=q^{\ell}$ where $q=e^{2 \pi i / n}$. This choice suggests the following generalization of Eqs. (4) and (5). 


$$
\begin{aligned}
& \tau^{n}=1, \\
& \tau^{\dagger}=\tau^{-1} .
\end{aligned}
$$

Note that for $n=2$, according to Eq. (4), $\tau^{-1}=\tau$ and Eq. (56) coincides with (5).

In the following we shall only consider $\mathbb{Z}_{n}$-graded TSs with one symmetry generator $\mathcal{Q}$. In order to proceed along the same lines as in the case of $n=2$, we need to impose a grading condition on $\mathcal{Q}$ similar to (11). We first consider the simplest case, namely, $\mathbb{Z}_{n}$-graded UTS of type $(1,1, \ldots, 1)$.

\section{1. $\mathbb{Z}_{n}$-graded UTS of type $(1,1, \ldots, 1)$}

For $n=2$, condition (11) implies that the action of $\mathcal{Q}$ on a definite color (parity) state vector changes its color (parity) - a bosonic state changes to a fermionic state and vice versa. The simplest generalization of this statement to the case $n>2$ is that the action of $\mathcal{Q}$ must change the color of a definite color state by one unit, i.e.,

$$
\tau \psi=c_{\ell} \psi \quad \text { implies } \quad \tau(\mathcal{Q} \psi)=c_{\ell+1} Q \psi .
$$

This condition is consistent with Eq. (55). If we impose this condition on the representations $\mathcal{Q}^{E}$ and $\tau^{E}$ in $\mathcal{H}_{E}$, then in a basis in which $\tau^{E}$ is diagonal we have

$$
\tau^{E}=\operatorname{diag}\left(q, q^{2}, \ldots, q^{n-1}, q^{n}=1\right),
$$

and

$$
\mathcal{Q}^{E}=\left(\begin{array}{ccccccc}
0 & 0 & 0 & \cdots & 0 & 0 & a_{n} \\
a_{1} & 0 & 0 & \cdots & 0 & 0 & 0 \\
0 & a_{2} & 0 & \cdots & 0 & 0 & 0 \\
\vdots & \vdots & \ddots & & \vdots & \vdots & \vdots \\
0 & 0 & 0 & \cdots & a_{n-2} & 0 & 0 \\
0 & 0 & 0 & \cdots & 0 & a_{n-1} & 0
\end{array}\right),
$$

where $a_{\ell}$ are complex numbers depending on $E$.

A simple calculation shows that $\tau^{E}$ and $\mathcal{Q}^{E} q$-commute, i.e., $\left[\tau^{E}, \mathcal{Q}^{E}\right]_{q}=0$, where the $q$-commutator is defined by $\left[O_{1}, O_{2}\right]_{q}:=O_{1} O_{2}-q O_{2} O_{1}$. Generalizing this property of $\tau^{E}$ and $\mathcal{Q}^{E}$ to $\tau$ and $\mathcal{Q}$, we find

$$
[\tau, \mathcal{Q}]_{q}=0 .
$$

This relation is the algebraic expression of the condition (57). It reduces to Eq. (11) for $n=2$.

Another consequence of Eq. (59) is that a symmetry generator of a $\mathbb{Z}_{n}$-graded UTS of type $(1,1, \ldots, 1)$ with $n>2$ which satisfies (57) cannot be self-adjoint. Furthermore, one can easily check that

$$
\left(\mathcal{Q}^{E}\right)^{n}=a_{1} a_{2} \cdots a_{n} I_{n \times n} .
$$


We can generalize this equation to the whole Hilbert space and write it in the operator form:

$$
\mathcal{Q}^{n}=\mathcal{K} .
$$

Here $\mathcal{K}$ is an operator that commutes with all other operators in the algebra.

Next, we seek for the algebraic relations satisfied by the self-adjoint generators:

$$
Q_{1}:=\frac{1}{\sqrt{2}}\left(\mathcal{Q}+\mathcal{Q}^{\dagger}\right) \quad \text { and } \quad Q_{2}:=\frac{-i}{\sqrt{2}}\left(\mathcal{Q}-\mathcal{Q}^{\dagger}\right) .
$$

Using the results reported in Appendix A, namely, Eq. (A.18), one can show that in an energy eigenspace $\mathcal{H}_{E}$, with $E>0, Q_{1}$ and $Q_{2}$ satisfy

$$
\begin{aligned}
& \left(Q_{1}^{E}\right)^{n}+\alpha_{n-2}\left(Q_{1}^{E}\right)^{n-2}+\cdots=\left(\frac{1}{\sqrt{2}}\right)^{n} R_{1}, \\
& \left(Q_{2}^{E}\right)^{n}+\alpha_{n-2}\left(Q_{2}^{E}\right)^{n-2}+\cdots=\left(\frac{1}{\sqrt{2}}\right)^{n} R_{2},
\end{aligned}
$$

where $\alpha_{\ell}$ 's are functions of $\left|a_{i}\right|^{2}$ and $R_{1}$ and $R_{2}$ are defined by

$$
R_{1}:=\prod_{k=1}^{n} a_{k}+\prod_{k=1}^{n} a_{k}^{*}, \quad R_{2}:=\prod_{k=1}^{n}\left(-i a_{k}\right)+\prod_{k=1}^{n}\left(i a_{k}^{*}\right) .
$$

Eqs. (64) and (65) can be written in the operator form according to

$$
\begin{aligned}
& Q_{1}^{n}+M_{n-2} Q_{1}^{n-2}+\cdots=\left(\frac{1}{\sqrt{2}}\right)^{n}\left(\mathcal{K}+\mathcal{K}^{\dagger}\right), \\
& Q_{2}^{n}+M_{n-2} Q_{2}^{n-2}+\cdots=\left(\frac{1}{\sqrt{2}}\right)^{n}\left(i^{n} \mathcal{K}^{\dagger}+(-i)^{n} \mathcal{K}\right),
\end{aligned}
$$

where $M_{i}$ 's are self-adjoint operators commuting with all other operators.

We can rewrite Eqs. (66) and (67) in the following more symmetric way.

For $n=2 p$,

$$
\begin{aligned}
& \left(Q_{1}^{2}-\mathcal{M}_{1}\right)\left(Q_{1}^{2}-\mathcal{M}_{2}\right) \cdots\left(Q_{1}^{2}-\mathcal{M}_{p}\right)=\left(\frac{1}{2}\right)^{p}\left(\mathcal{K}+\mathcal{K}^{\dagger}\right), \\
& \left(Q_{2}^{2}-\mathcal{M}_{1}\right)\left(Q_{2}^{2}-\mathcal{M}_{2}\right) \cdots\left(Q_{2}^{2}-\mathcal{M}_{p}\right)=\left(\frac{-1}{2}\right)^{p}\left(\mathcal{K}+\mathcal{K}^{\dagger}\right) .
\end{aligned}
$$

For $n=2 p+1$,

$$
\begin{aligned}
& \left(Q_{1}^{2}-\mathcal{M}_{1}\right)\left(Q_{1}^{2}-\mathcal{M}_{2}\right) \cdots\left(Q_{1}^{2}-\mathcal{M}_{p}\right) Q_{1}=\left(\frac{1}{\sqrt{2}}\right)^{2 p+1}\left(\mathcal{K}+\mathcal{K}^{\dagger}\right), \\
& \left(Q_{2}^{2}-\mathcal{M}_{1}\right)\left(Q_{2}^{2}-\mathcal{M}_{2}\right) \cdots\left(Q_{2}^{2}-\mathcal{M}_{p}\right) Q_{2}=\left(\frac{i}{\sqrt{2}}\right)^{2 p+1}\left(\mathcal{K}^{\dagger}-\mathcal{K}\right) .
\end{aligned}
$$

Here $\mathcal{M}_{i}$ are also operators that commute with all other operators. Note also that for even $p$ 's, the algebraic relations for $Q_{1}$ and $Q_{2}$ coincide.

Eqs. (62), (69), and (70) are the defining equations of the algebra of $\mathbb{Z}_{n}$-graded TS of type $(1,1, \ldots, 1)$. For $n=2$, they reduce to the familiar algebra of $\mathbb{Z}_{2}$-graded TS 
of type $(1,1)$. In this case, as we discussed in Subsection 3.1, we can perform a linear transformation on the self-adjoint generators that eliminates the operator $\mathcal{K}$.

Next, consider the case where for all $E>0, \mathcal{K}^{E} \neq 0$ and introduce the transformed generator $\widetilde{\mathcal{Q}}$ by

$$
\widetilde{\mathcal{Q}}^{E}=\left(\frac{E}{\left|\kappa^{E}\right|}\right)^{1 / n} e^{-i \phi^{E} / n} \mathcal{Q}^{E},
$$

where

$$
\kappa^{E}:=a_{1} a_{2} \cdots a_{n} \quad \text { and } \quad e^{i \phi^{E}}:=\frac{\kappa^{E}}{\left|\kappa^{E}\right|} .
$$

In view of Eqs. (61) and (70), it is not difficult to see that

$$
\left(\widetilde{\mathcal{Q}}^{E}\right)^{n}=E I_{n},
$$

Writing this equation in the operator form, we are led to

$$
H=\widetilde{\mathcal{Q}}^{n} .
$$

This is precisely the algebra of fractional supersymmetry of order $n[10,11]$.

Next, we examine whether Eq. (62) guarantees the desired degeneracy structure of the $\mathbb{Z}_{n}$-graded TS of type $(1,1, \ldots, 1)$. In order to address this question, we suppose that the kernel of $\mathcal{K}$ is a subset of $\mathcal{H}_{0}$. Then for all $E>0, \kappa^{E} \neq 0$.

In view of Eq. (62), the eigenvalues of $\mathcal{Q}^{E}$ are of the form $q^{\ell}\left(\kappa^{E}\right)^{1 / n}$, with $\ell \in$ $\{1,2, \ldots, n\}$. Now, let $\left|q^{\ell}, v_{\ell}\right\rangle$ denote the corresponding eigenvectors, where $v_{\ell}$ 's are degeneracy labels. We can easily show using Eq. (60) that for all positive integers $s$, $\tau^{s}\left|q^{\ell}, v_{\ell}\right\rangle$ are eigenvectors of $\mathcal{Q}^{E}$ with eigenvalue $q^{\ell-s}\left(\kappa^{E}\right)^{1 / n}$. This is sufficient to conclude that all the eigenvalues of $\mathcal{Q}^{E}$ are either nondegenerate or have the same multiplicity $N_{E}$. If for all $E>0, N_{E}=1$, then we will have the desired degeneracy structure of the $\mathbb{Z}_{n}$-graded UTS of type $(1,1, \ldots, 1)$. If there are $E>0$ for which $N_{E}>1$, then we have a nonuniform $\mathbb{Z}_{n}$-graded TS of type $(1,1, \ldots, 1)$.

We conclude this section by noting that if we put $\mathcal{K}=0$ in the algebraic relations for $\mathbb{Z}_{n}$-graded UTS of type $(1,1, \ldots, 1)$, we will obtain the algebraic relations for $\mathbb{Z}_{2}$-graded TS. This is not so strange, because putting $\mathcal{K}=0$ means that one of the $a_{i}$ 's (say $a_{n}$ ) in $\mathcal{Q}^{E}$ is zero. In this case, as we explain in Appendix A, there is a unitary transformation that transforms $Q_{1}^{E}$ and $Q_{2}^{E}$ to off block diagonal matrices. In view of the analysis of Section 3, this implies that the generators of this kind of $\mathbb{Z}_{n}$-graded UTSs satisfy the algebra of $\mathbb{Z}_{2}$-graded UT.

\section{2. $\mathbb{Z}_{n}$-graded TS of arbitrary type $\left(m_{1}, m_{2}, \ldots, m_{n}\right)$}

In order to obtain the algebraic structure of the $\mathbb{Z}_{n}$-graded TS of arbitrary type $\left(m_{1}, m_{2}, \ldots, m_{n}\right)$, we need an appropriate grading operator. We shall adopt Eqs. (6), (55), (56) and (60) as the defining conditions for our $\mathbb{Z}_{n}$-grading operator. We shall further assume that $m_{1} \leqslant m_{2} \leqslant \cdots \leqslant m_{n}$. This ordering can always be achieved by a reassignment of the colors. Again we shall confine our attention to the uniform $\mathbb{Z}_{n}$-graded TS. The algebras of uniform and nonuniform TS of the same type are identical. 
Working in an eigenbasis in which $\tau^{E}$ is diagonal and using Eq. (60), we have

$$
\tau^{E}=\operatorname{diag}(\underbrace{q, q, \ldots, q}_{m_{1} \text { times }}, \underbrace{q^{2}, q^{2}, \ldots, q^{2}}_{m_{2} \text { times }}, \ldots, \underbrace{q^{n}, q^{n}, \ldots, q^{n}}_{m_{n} \text { times }}) .
$$

In view of this equation and Eq. (60), we obtain the following matrix representation for $\mathcal{Q}^{E}$.

$$
\mathcal{Q}^{E}=\left(\begin{array}{ccccccc}
0 & 0 & 0 & \cdots & 0 & 0 & A_{n} \\
A_{1} & 0 & 0 & \cdots & 0 & 0 & 0 \\
0 & A_{2} & 0 & \cdots & 0 & 0 & 0 \\
\vdots & \vdots & \ddots & & \vdots & \vdots & \vdots \\
0 & 0 & 0 & \cdots & A_{n-2} & 0 & 0 \\
0 & 0 & 0 & \cdots & 0 & A_{n-1} & 0
\end{array}\right),
$$

where $A_{\ell}$ (with $\ell \in\{1,2, \ldots, n-1\}$ ) are complex $m_{\ell+1} \times m_{\ell}$ matrices, $A_{n}$ is a complex $m_{1} \times m_{n}$ matrix, and 0's are appropriate zero matrices.

Next, we compute the $n$th power of $\mathcal{Q}$. The result is

$$
\left(\mathcal{Q}^{E}\right)^{n}=\left(\begin{array}{ccccc}
A_{n} A_{n-1} \cdots A_{2} A_{1} & 0 & 0 & \cdots & 0 \\
0 & A_{1} A_{n} A_{n-1} \cdots A_{2} & 0 & \cdots & 0 \\
\vdots & \vdots & \ddots & \vdots & \vdots \\
0 & 0 & 0 & \cdots & A_{n-1} A_{n-2} \cdots A_{1} A_{n}
\end{array}\right) .
$$

In order to find the most general algebraic identity satisfied by $\mathcal{Q}$ we appeal to the following generalization of Lemma 1. The proof is given in Appendix A.

Lemma 2. Let $\left(m_{1}, m_{2}, \ldots, m_{n}\right)$ be an $n$-tuple of positive integers satisfying $m_{1} \leqslant m_{2} \leqslant$ $\ldots \leqslant m_{n}, m:=\sum_{\ell=1}^{n} m_{\ell}, \delta$ is the number of times $m_{1}$ appears in $\left(m_{1}, m_{2}, \ldots, m_{n}\right), Q$ is an $m \times m$ matrix of the form (75), and $\mathcal{P}(x)$ is the characteristic polynomial of the $m_{1} \times m_{1}$ matrix $A_{n} A_{n-1} \cdots A_{2} A_{1}$. Then $Q$ satisfies

$$
\mathcal{P}\left(Q^{n}\right) Q^{n-\delta}=0 \text {. }
$$

Substituting $\mathcal{Q}^{E}$ for $Q$ in Eq. (77) and writing $\mathcal{P}(x)$ in terms of its roots $\kappa_{k}^{E}$, we find

$$
\left[\left(\mathcal{Q}^{E}\right)^{n}-\kappa_{1}^{E}\right]\left[\left(\mathcal{Q}^{E}\right)^{n}-\kappa_{2}^{E}\right] \cdots\left[\left(\mathcal{Q}^{E}\right)^{n}-\kappa_{m_{1}}^{E}\right]\left(\mathcal{Q}^{E}\right)^{n-\delta}=0 .
$$

Next, we introduce the operators $\mathcal{K}_{k}$ for $k \in\left\{1,2, \ldots, m_{1}\right\}$ which commute with the Hamiltonian and have the representation:

$$
\mathcal{K}_{k}^{E}=\kappa_{k}^{E} I_{m}
$$

in $\mathcal{H}_{E}$. In view of Eqs. (78) and (79), we obtain

$$
\left(\mathcal{Q}^{n}-\mathcal{K}_{1}\right)\left(\mathcal{Q}^{n}-\mathcal{K}_{2}\right) \cdots\left(\mathcal{Q}^{n}-\mathcal{K}_{m_{1}}\right) \mathcal{Q}^{n-\delta}=0 .
$$


The operators $\mathcal{K}_{k}$ have a similar degeneracy structure as the Hamiltonian (at least for the positive energy eigenvalues). Therefore, the Hamiltonian might be expressed as a function of $\mathcal{K}_{k}$.

For a $\mathbb{Z}_{n}$-graded UTS of type $(1,1, \ldots, 1), \delta=n$ and Eq. (80) reduces to (62). However, one can show that in general Eq. (80) does not ensure the desired degeneracy structure of the general $\mathbb{Z}_{n}$-graded TSs. This is true even for the case $n=2, m_{+}=2, m_{-}=1$ considered in Section 3. In general, the $\mathbb{Z}_{n}$-graded TSs correspond to a special class of symmetries satisfying (80).

Finally, we wish to note that for a general $\mathbb{Z}_{n}$-graded TS the algebraic relations satisfied by the self-adjoint generators are extremely complicated. We have not been able to express them in a closed form.

\section{Mathematical interpretation of $\boldsymbol{\Delta}_{i, j}$}

In order to obtain the mathematical interpretation of the invariants $\Delta_{i, j}$ of TSs, one must express the Hamiltonian in terms of the symmetry generators. This can be easily done using the defining algebra for the $\mathbb{Z}_{n}$-graded TSs of type $(1,1, \ldots, 1)$. In the following, we discuss the mathematical meaning of the topological invariants associated with these symmetries.

We know that for a $\mathbb{Z}_{n}$-graded TS of type $(1,1, \ldots, 1)$ the operator $\mathcal{K}$ has a similar degeneracy structure as the Hamiltonian. Therefore, we may set $H=f(\mathcal{K})$ where $f$ is a function mapping the eigenvalues $\kappa^{E}$ to $E$. Next, suppose that the kernels of $\mathcal{K}$ and $H$ also coincide. Then as far as the general properties of the symmetry is concerned, we can confine our attention to the special case where $\mathcal{K}=H$, i.e., the fractional supersymmetry. Note that in this case, $\mathcal{Q}^{n}$ is necessarily self-adjoint. Alternatively, we can use the rescaled symmetry generator $\widetilde{\mathcal{Q}}$ that does satisfy $\widetilde{\mathcal{Q}}^{n}=H$.

In order to obtain the mathematical interpretation of the topological invariants $\Delta_{i, j}$, we use an $n$-component representation of the Hilbert space in which a state vector $\psi$ is represented by a column of $n$ colored vectors $\psi_{\ell} \in \mathcal{H}_{\ell}$. In this representation the grading operator is diagonal and the generator of the symmetry and the Hamiltonian are, respectively, expressed by $n \times n$ matrices of operators according to

$$
\mathcal{Q}=\left(\begin{array}{ccccccc}
0 & 0 & 0 & \cdots & 0 & 0 & D_{n} \\
D_{1} & 0 & 0 & \cdots & 0 & 0 & 0 \\
0 & D_{2} & 0 & \cdots & 0 & 0 & 0 \\
\vdots & \ldots & \ddots & & \vdots & \vdots & \vdots \\
0 & 0 & 0 & \cdots & D_{n-2} & 0 & 0 \\
0 & 0 & 0 & \cdots & 0 & D_{n-1} & 0
\end{array}\right),
$$




$$
H=\mathcal{Q}^{n}=\left(\begin{array}{cccccc}
H_{1} & 0 & 0 & \cdots & 0 & 0 \\
0 & H_{2} & 0 & \cdots & 0 & 0 \\
\vdots & \vdots & \ddots & \cdots & \vdots & \vdots \\
0 & 0 & 0 & \cdots & 0 & H_{n}
\end{array}\right) .
$$

Here $D_{n}: \mathcal{H}_{n} \rightarrow \mathcal{H}_{1}$ and $D_{\ell}: \mathcal{H}_{\ell} \rightarrow \mathcal{H}_{\ell+1}$, with $\ell \in\{1,2, \ldots, n-1\}$, are operators and

$$
\begin{aligned}
& H_{1}:=D_{n} D_{n-1} \cdots D_{2} D_{1}, \\
& H_{2}:=D_{1} D_{n} D_{n-1} \cdots D_{2}, \\
& \cdots \quad \cdots \\
& H_{n}:=D_{n-1} D_{n-2} \cdots D_{1} D_{n} .
\end{aligned}
$$

The condition that $H$ is self-adjoint takes the form $H_{\ell}^{\dagger}=H_{\ell}$, or alternatively

$$
D_{\sigma(n)} D_{\sigma(n-1)} \cdots D_{\sigma(2)} D_{\sigma(1)}=D_{\sigma(1)}^{\dagger} D_{\sigma(2)}^{\dagger} \cdots D_{\sigma(n-1)}^{\dagger} D_{\sigma(n)}^{\dagger},
$$

for all cyclic permutations $\sigma$ of $(n, n-1, n-2, \ldots, 1)$. In addition, the assumption that $H$ has a nonnegative spectrum further restricts $D_{\ell}$. Note also that the algebraic relations satisfied by the self-adjoint generators also put restrictions on the choice of the operators $D_{\ell}$. This is because the operators $M_{i}$ appearing in Eqs. (66) and (67) involve $D_{\ell}$. The condition that $M_{i}$ commute with $\mathcal{Q}$ leads to a set of compatibility relations among $D_{\ell}$.

In view of Eq. (82) and the fact that $n_{\ell}^{(0)}$ is the dimension of the kernel of $H_{\ell}$, we can easily express the invariant $\Delta_{i j}$ in the form:

$$
\Delta_{i, j}=\operatorname{dim}\left(\operatorname{ker} H_{j}\right)-\operatorname{dim}\left(\operatorname{ker} H_{i}\right) .
$$

Suppose that the subspaces $\mathcal{H}_{\ell}$ are all identified with a fixed Hilbert Space. Consider the special case where

$$
D_{3}=D_{4}=\cdots=D_{n}=1, \quad D_{2}=D_{1}^{\dagger},
$$

and $D_{1}$ is a Fredholm operator, then $H_{1}=D_{1}^{\dagger} D_{1}, H_{2}=D_{1} D_{1}^{\dagger}$, and there is one independent invariant, namely

$$
\Delta_{1,2}=\operatorname{dim}\left(\operatorname{ker} D_{1}^{\dagger} D_{1}\right)-\operatorname{dim}\left(\operatorname{ker} D_{1} D_{1}^{\dagger}\right)=\operatorname{dim}\left(\operatorname{ker} D_{1}\right)-\operatorname{dim}\left(\operatorname{ker} D_{1}^{\dagger}\right) .
$$

This is just the analytic index of $D_{1}$. This example shows that the above construction has nontrivial solutions.

In general, the operator $D_{\ell}$ need not satisfy (85). They are however subject to the abovementioned compatibility relations. In order to demonstrate the nature of these relations, we consider $\mathbb{Z}_{3}$-graded UTS of type $(1,1,1)$ with the symmetry generator

$$
\mathcal{Q}=\left(\begin{array}{ccc}
0 & 0 & D_{3} \\
D_{1} & 0 & 0 \\
0 & D_{2} & 0
\end{array}\right) .
$$

In this case, the algebraic relations (66) and (67) satisfied by the self-adjoint generators involve a single commuting operator which we denote by $M$. Assuming that this operator has the form 


$$
M=\frac{1}{2}\left(\begin{array}{ccc}
M_{1} & 0 & 0 \\
0 & M_{2} & 0 \\
0 & 0 & M_{3}
\end{array}\right),
$$

and enforcing

$$
[\mathcal{Q}, M]=0, \quad Q_{1}^{3}+M Q_{1}=\frac{1}{\sqrt{2}} H,
$$

we find

$$
\begin{aligned}
& M_{1} D_{3}=D_{3} M_{3}, \\
& M_{2} D_{1}=D_{1} M_{1}, \\
& M_{3} D_{2}=D_{2} M_{2} .
\end{aligned}
$$

One can manipulate these relations to obtain the following compatibility relations for $D_{\ell}$.

$$
\begin{aligned}
& D_{2}^{\dagger} D_{2} D_{1} D_{3}=D_{1} D_{3} D_{2} D_{2}^{\dagger}, \\
& D_{3}^{\dagger} D_{3} D_{2} D_{1}=D_{2} D_{1} D_{3} D_{3}^{\dagger}, \\
& D_{1}^{\dagger} D_{1} D_{3} D_{2}=D_{3} D_{2} D_{1} D_{1}^{\dagger} .
\end{aligned}
$$

Furthermore, under these conditions on $M_{\ell}$ and $D_{\ell}$, one can check that the relation for $Q_{2}$, i.e., $Q_{2}^{3}+M Q_{2}=0$, is identically satisfied.

Next, consider the case where one of the $D_{i}$ 's, say $D_{3}$, is 1 . Then we can use Eqs. (89)(91) to express $M_{i}$ in terms of $D_{1}$ and $D_{2}$. This yields

$$
M_{1}=M_{3}=-\left(D_{1}^{\dagger} D_{1}+D_{2} D_{2}^{\dagger}+1\right), \quad M_{2}=-\left(D_{2}^{\dagger} D_{2}+D_{1} D_{1}^{\dagger}+1\right) .
$$

Note that we can easily satisfy Eqs. (92)-(94), if $D_{2}=D_{1}^{\dagger}$, i.e., when (85) is satisfied. In this case,

$$
\begin{aligned}
& M_{1}=M_{2}=-\left(2 D_{1}^{\dagger} D_{1}+1\right)=-\left(2 H_{1}+1\right), \\
& M_{3}=-\left(2 D_{1} D_{1}^{\dagger}+1\right)=-\left(2 H_{2}+1\right),
\end{aligned}
$$

and

$$
M=-\left(H+\frac{1}{2}\right),
$$

where we have used Eqs. (82) and (87).

\section{Examples}

In this section, we examine some examples of quantum systems possessing UTSs. 


\subsection{A system with UTS of type $(2,1)$}

Consider the Hamiltonian

$$
H=\frac{1}{2}\left(p^{2}+x^{2}\right)+\frac{1}{2}\left(\begin{array}{ccc}
1 & 0 & 0 \\
0 & 1 & 0 \\
0 & 0 & -1
\end{array}\right)
$$

where $x$ and $p$ are, respectively, the position and momentum operators. This Hamiltonian was originally considered in [8]. It is not difficult to show that it commutes with

$$
\mathcal{Q}=\left(\begin{array}{ccc}
0 & 0 & \frac{1}{\sqrt{2}}(p-i x) \\
0 & 0 & 0 \\
0 & \frac{1}{\sqrt{2}}(p+i x) & 0
\end{array}\right) .
$$

Furthermore, $\mathcal{Q}, H$, and the the self-adjoint generators $Q_{j}$ satisfy the algebra of the UTS of type $(2,1)$, i.e.,

$$
Q_{1}^{3}=H Q_{1}, \quad Q_{2}^{3}=H Q_{2}, \quad \mathcal{Q}^{3}=0 .
$$

For this system the grading operator is given by $\tau=\operatorname{diag}(1,1,-1)$; there is a nondegenerate zero-energy ground state; and the positive energy eigenvalues are triply degenerate. Therefore, this system has a UTS of type $(2,1)$.

If we denote by $|n\rangle$ the normalized energy eigenvectors of the harmonic oscillator with unit mass and frequency, then a set of eigenvectors of the Hamiltonian (98) are given by

$$
\left|\phi_{0}\right\rangle=\left(\begin{array}{c}
0 \\
0 \\
|0\rangle
\end{array}\right)
$$

for $E=0$, and

$$
\left|\phi_{n}, 1\right\rangle=\left(\begin{array}{c}
|n-1\rangle \\
0 \\
0
\end{array}\right), \quad\left|\phi_{n}, 2\right\rangle=\left(\begin{array}{c}
0 \\
|n-1\rangle \\
0
\end{array}\right), \quad\left|\phi_{n}, 3\right\rangle=\left(\begin{array}{c}
0 \\
0 \\
|n\rangle
\end{array}\right),
$$

for $E=n>0$.

Next, we check the action of the symmetry generator and the grading operator on $\left|\phi_{0}\right\rangle$ and $\left|\phi_{n}, a\right\rangle$. This yields

$$
\begin{aligned}
& \mathcal{Q}\left|\phi_{0}\right\rangle=0, \quad \tau\left|\phi_{0}\right\rangle=-\left|\phi_{0}\right\rangle, \\
& \mathcal{Q}\left|\phi_{n}, 1\right\rangle=0, \quad \mathcal{Q}\left|\phi_{n}, 2\right\rangle \rightarrow\left|\phi_{n}, 3\right\rangle, \quad \mathcal{Q}\left|\phi_{n}, 3\right\rangle \rightarrow\left|\phi_{n}, 1\right\rangle, \\
& \tau\left|\phi_{n}, 1\right\rangle=\left|\phi_{n}, 1\right\rangle, \quad \tau\left|\phi_{n}, 2\right\rangle=\left|\phi_{n}, 2\right\rangle, \quad \tau\left|\phi_{n}, 3\right\rangle=-\left|\phi_{n}, 3\right\rangle .
\end{aligned}
$$

Here we have used $\rightarrow$ to denote equality up to a nonzero multiplicative constant.

As one can see from Eqs. (102) and (104), the ground state has negative parity and the positive energy levels consist of two positive and one negative parity states. The topological invariants for this system are $\Delta_{2,1}=-\Delta_{1,2}=-2$. 


\subsection{A system with UTS of type $(1,1,1)$}

Consider the Hamiltonian

$$
H=\mathcal{Q}^{3}=\frac{1}{2}\left(p^{2}+x^{2}\right)+\frac{1}{2}\left(\begin{array}{ccc}
1 & 0 & 0 \\
0 & -1 & 0 \\
0 & 0 & 1
\end{array}\right)
$$

which is precisely the Hamiltonian (98) written in another basis. ${ }^{4}$ It possesses a symmetry generated by

$$
\mathcal{Q}=\left(\begin{array}{ccc}
0 & 0 & 1 \\
\frac{1}{\sqrt{2}}(p+i x) & 0 & 0 \\
0 & \frac{1}{\sqrt{2}}(p-i x) & 0
\end{array}\right) .
$$

In fact, it is not difficult to show that

$$
\mathcal{Q}^{3}=H \text {. }
$$

Furthermore, one can check that the self-adjoint generators $Q_{j}$ satisfy

$$
Q_{1}^{3}+M Q_{1}=\frac{1}{\sqrt{2}} H, \quad Q_{2}^{3}+M Q_{2}=0,
$$

where the operator $M$ is given by

$$
M=-\frac{1}{2}\left(p^{2}+x^{2}\right)+\left(\begin{array}{ccc}
-1 & 0 & 0 \\
0 & 0 & 0 \\
0 & 0 & -1
\end{array}\right)=-\left(H+\frac{1}{2}\right) .
$$

This is in agreement with the more general treatment of Section 5. In particular, Eq. (109) is a special case of Eq. (97).

Obviously, $M$ commutes with $H$ and $\mathcal{Q}$. In view of this observation and Eqs. (107), (108), (62), (66), and (67) we can conclude that this system has a $\mathbb{Z}_{3}$-graded UTS of type $(1,1,1)$.

A complete set of eigenvectors of the Hamiltonian (98) are given by

$$
\left|\phi_{0}\right\rangle=\left(\begin{array}{c}
0 \\
|0\rangle \\
0
\end{array}\right)
$$

for $E=0$, and

$$
\left|\phi_{n}, 1\right\rangle=\left(\begin{array}{c}
|n-1\rangle \\
0 \\
0
\end{array}\right), \quad\left|\phi_{n}, 2\right\rangle=\left(\begin{array}{c}
0 \\
|n\rangle \\
0
\end{array}\right), \quad\left|\phi_{n}, 3\right\rangle=\left(\begin{array}{c}
0 \\
0 \\
|n-1\rangle
\end{array}\right),
$$

for $E=n>0$.

\footnotetext{
${ }^{4}$ We have used some of the results of [11] to obtain this Hamiltonian.
} 
The grading operator is $\tau=\operatorname{diag}\left(q, q^{2}, 1\right)$, where $q:=e^{2 \pi i / 3}$. The symmetry generator $\mathcal{Q}$ and the grading operator $\tau$ transform the energy eigenvectors according to

$$
\begin{array}{lll}
\mathcal{Q}\left|\phi_{0}\right\rangle=0, & \tau\left|\phi_{0}\right\rangle=q^{2}\left|\phi_{0}\right\rangle, & \\
\mathcal{Q}\left|\phi_{n}, 1\right\rangle \rightarrow\left|\phi_{n}, 2\right\rangle, & \mathcal{Q}\left|\phi_{n}, 2\right\rangle \rightarrow\left|\phi_{n}, 3\right\rangle, & \mathcal{Q}\left|\phi_{n}, 3\right\rangle \rightarrow\left|\phi_{n}, 1\right\rangle, \\
\tau\left|\phi_{n}, 1\right\rangle=q\left|\phi_{n}, 1\right\rangle, & \tau\left|\phi_{n}, 2\right\rangle=q^{2}\left|\phi_{n}, 2\right\rangle, & \tau\left|\phi_{n}, 3\right\rangle=\left|\phi_{n}, 3\right\rangle .
\end{array}
$$

In particular, $\left|\phi_{0}\right\rangle$ and $\left|\phi_{n}, a\right\rangle$ have colors $q^{2}$ and $q^{a}$, respectively, and the topological invariants of the system are given by

$$
\Delta_{1,2}=-\Delta_{2,1}=1, \quad \Delta_{2,3}=-\Delta_{3,2}=-1, \quad \Delta_{1,3}=-\Delta_{3,1}=0 .
$$

\subsection{A system with UTS of type $(1,1, \ldots, 1)$}

Consider the Hamiltonian

$$
H=\frac{1}{2}\left(p^{2}+x^{2}\right)+\frac{1}{2} \operatorname{diag}(\underbrace{1,1, \ldots, 1}_{(n-1) \text {-times }},-1) .
$$

This Hamiltonian has a symmetry generated by

$$
\mathcal{Q}=\left(\begin{array}{ccccc}
0 & 0 & \cdots & 0 & \frac{1}{\sqrt{2}}(p-i x) \\
1 & 0 & \cdots & 0 & 0 \\
0 & 1 & \cdots & 0 & 0 \\
\vdots & \vdots & \ddots & \vdots & \vdots \\
0 & 0 & \cdots & \frac{1}{\sqrt{2}}(p+i x) & 0
\end{array}\right),
$$

because $\mathcal{Q}^{n}=H$.

One can also check that the self-adjoint generators $Q_{j}$ satisfy

$$
\begin{aligned}
& Q_{1}^{n}+M_{n-2} Q_{1}^{n-2}+\cdots=\left(\frac{1}{\sqrt{2}}\right)^{n}(2 H), \\
& Q_{2}^{n}+M_{n-2} Q_{2}^{n-2}+\cdots=\left(\frac{i}{\sqrt{2}}\right)^{n}\left(1+(-1)^{n}\right) H,
\end{aligned}
$$

where $M_{n-2 k}$ are given by

$$
M_{n-2 k}=(-1)^{k}\left[\frac{1}{2^{k}}\left(\begin{array}{c}
n-k-1 \\
k
\end{array}\right)+\frac{1}{2^{k-1}}\left(\begin{array}{c}
n-k-1 \\
k-1
\end{array}\right) H\right],
$$

and $\left(\begin{array}{l}a \\ b\end{array}\right):=\frac{a !}{b !(a-b) !}$.

It is not difficult to see that this system has a zero-energy ground state and that the positive energy levels are $n$-fold degenerate. A complete set of energy eigenvectors are given by 


$$
|0\rangle=\left(\begin{array}{c}
0 \\
0 \\
\vdots \\
0 \\
|0\rangle
\end{array}\right)
$$

for $E=0$, and

$$
|m, 1\rangle=\left(\begin{array}{c}
|m-1\rangle \\
0 \\
\vdots \\
0 \\
0
\end{array}\right), \ldots, \quad|m, n-1\rangle=\left(\begin{array}{c}
0 \\
0 \\
\vdots \\
|m-1\rangle \\
0
\end{array}\right), \quad|m, n\rangle=\left(\begin{array}{c}
0 \\
0 \\
\vdots \\
0 \\
|m\rangle
\end{array}\right) \text {, }
$$

for $E=m>0$ :

These observations indicate that the quantum system defined by the Hamiltonian (116) has a $\mathbb{Z}_{n}$-graded UTS of type $(1,1, \ldots, 1)$. Clearly, the grading operator is $\tau=$ $\operatorname{diag}\left(q, q^{2}, \ldots, q^{n-1}, q^{n}=1\right)$, the ground state has color $c_{n}=q^{n}=1$, and the nonvanishing topological invariants are $\Delta_{\ell, n}=-\Delta_{n, \ell}=1$, where $\ell \in\{1,2, \ldots, n-1\}$.

Next, we wish to comment that if we change the sign of the term involving the matrix $\operatorname{diag}(\underbrace{1,1, \ldots, 1}_{(n-1) \text {-times }},-1)$ in the Hamiltonian $(116)$, then we obtain another quantum system with a $\mathbb{Z}_{n}$-graded UTS of type $(1,1, \ldots, 1)$ that is generated by

$$
\mathcal{Q}=\left(\begin{array}{ccccc}
0 & 0 & \cdots & 0 & \frac{1}{\sqrt{2}}(p+i x) \\
1 & 0 & \cdots & 0 & 0 \\
0 & 1 & \cdots & 0 & 0 \\
\vdots & \vdots & \ddots & \vdots & \vdots \\
0 & 0 & \cdots & \frac{1}{\sqrt{2}}(p-i x) & 0
\end{array}\right),
$$

This system has an $(n-1)$-fold degenerate zero-energy ground state.

\section{Conclusions}

We have introduced a generalization of supersymmetry that shares its topological properties. We gave a complete description of the underlying algebraic structure and commented on the meaning of the corresponding topological invariants.

We showed that the algebras of the $\mathbb{Z}_{2}$-graded TSs of type $\left(m_{+}, 1\right)$ coincide with the algebras of supersymmetry or parasupersymmetry of order $p=2$. The algebraic relations obtained for the $\mathbb{Z}_{2}$-graded TSs of type $\left(m_{+}, m_{-}\right)$with $m_{-}>1$ include as special cases the algebras of higher order parasupersymmetry advocated by Durand and Vinet [16]. We also pointed out that the algebra of $\mathbb{Z}_{n}$-graded TS of type $(1,1, \ldots, 1)$ is related to the algebra of fractional supersymmetry of order $n$. 
Our approach in developing the concept of a TS differs from those of the other generalizations of supersymmetry in the sense that we introduce TSs in terms of certain requirements on the spectral degeneracy properties of the corresponding quantum systems, whereas in the other generalizations of supersymmetry such as parasupersymmetry and fractional supersymmetry one starts with certain defining algebraic relations. These relations are usually obtained by generalizing the relations satisfied by the generators of symmetries that relate degrees of freedom with different statistical properties in certain simple models. For example the Rubakov-Spiridonov algebra of parasupersymmetry of order $p=2$ was originally obtained by generalizing the algebra of symmetry generators of an oscillator involving a bosonic and a $(p=2)$ parafermionic degree of freedom [7]. In order to investigate the topological content of these (statistical) generalizations of supersymmetry, one is forced to study the spectral degeneracy structure of the corresponding systems. The derivation of the degeneracy structure using the defining algebraic relations is usually a difficult task. In fact, for parasupersymmetries of order $p>2$ this problem has not yet been solved. Even for the parasupersymmetries of order $p=2$ the solution requires a quite lengthy analysis [15], and the defining algebra does not guarantee the existence of any topological invariants. This in turn raises the question of the classification of the $p=2$ parasupersymmetries that do have topological properties similar to supersymmetry [13]. The analysis of the topological aspects of supersymmetry and $p=2$ parasupersymmetry shows that the information about the topological properties is contained in the spectral degeneracy structure of the corresponding systems. This is the main justification for our definition of a TS.

Like any other quantum mechanical symmetry, a TS also possesses an underlying operator algebra. This algebra contains more practical information about the systems possessing the symmetry. As we showed in the preceding sections, the operator algebras associated with TSs can be obtained using the defining conditions on the spectral degeneracy structure of these systems. This observation may be viewed as another indication that, as far as the topological aspects are concerned, the spectral degeneracy structure is more basic than the algebraic structure.

\section{Acknowledgements}

A.M. wishes to thank Bryce DeWitt for introducing him to supersymmetric quantum mechanics and encouraging him to work on the topological aspects of supersymmetry more than ten years ago. We would also like to thank M. Khorrami and F. Loran for interesting discussions and fruitful comments.

\section{Appendix A}

In this appendix we give the proofs of some of the mathematical results we use in Sections 3 and 4. In the following we shall denote the characteristic polynomial of a matrix $M$ by $\mathcal{P}_{M}(x)$, i.e., $\mathcal{P}_{M}(x)=\operatorname{det}(x I-M)$. 
Lemma 0. Let $X$ and $Y$ be $m \times n$ and $n \times m$ matrices respectively. Then

$$
\mathcal{P}_{Y X}(\lambda)=\lambda^{n-m} \mathcal{P}_{X Y}(\lambda) \text {. }
$$

Proof. Let

$$
M:=\left(\begin{array}{cc}
I_{m} & X \\
Y & \lambda I_{n}
\end{array}\right) .
$$

Then using the well-known properties of the determinant, we have

$$
\begin{aligned}
\operatorname{det}(M) & =\operatorname{det}\left(\begin{array}{cc}
I_{m} & X \\
Y & \lambda I_{n}
\end{array}\right)=\operatorname{det}\left(\begin{array}{cc}
I_{m} & X \\
0 & \lambda I_{n}-Y X
\end{array}\right) \\
& =\operatorname{det}\left(\lambda I_{n}-Y X\right)=\mathcal{P}_{Y X}(\lambda) .
\end{aligned}
$$

Similarly, we can show that

$$
\begin{aligned}
\operatorname{det}(M) & =\operatorname{det}\left(\begin{array}{cc}
I_{m} & X \\
Y & \lambda I_{n}
\end{array}\right)=\lambda^{n} \operatorname{det}\left(\begin{array}{cc}
I_{m} & X \\
\frac{1}{\lambda} Y & I_{n}
\end{array}\right) \\
& =\lambda^{n-m} \operatorname{det}\left(\begin{array}{cc}
\lambda I_{m} & \lambda X \\
\frac{1}{\lambda} Y & I_{n}
\end{array}\right)=\lambda^{n-m} \operatorname{det}\left(\begin{array}{cc}
\lambda I_{m}-X Y & 0 \\
\frac{1}{\lambda} Y & I_{n}
\end{array}\right) \\
& =\lambda^{n-m} \operatorname{det}\left(\lambda I_{m}-X Y\right)=\lambda^{n-m} \mathcal{P}_{X Y}(\lambda) .
\end{aligned}
$$

Eqs. (A.3) and (A.4) yield the identity (A.1).

Lemma 1. Let $m_{ \pm}$be positive integers, $m=m_{+}+m_{-}$, and $Q$ be an $m \times m$ matrix of the form

$$
Q=\left(\begin{array}{ll}
0 & X \\
Y & 0
\end{array}\right),
$$

where $X$ and $Y$ are $m_{+} \times m_{-}$and $m_{-} \times m_{+}$complex matrices. Then $\mathcal{P}_{X Y}\left(Q^{2}\right) Q=$ $\mathcal{P}_{Y X}\left(Q^{2}\right) Q=0$. Furthermore, if $m_{+}=m_{-}$, then $\mathcal{P}_{X Y}\left(Q^{2}\right)=\mathcal{P}_{Y X}\left(Q^{2}\right)=0$.

Proof. Let $a_{k}$ denote the coefficients of $\mathcal{P}_{Y X}(x)$, i.e., $\mathcal{P}_{Y X}(x)=\sum_{k=0}^{n} a_{k} x^{k}$. According to the Cayley-Hamilton theorem,

$$
\mathcal{P}_{Y X}(Y X)=\sum_{k=0}^{n} a_{k}(Y X)^{k}=0 .
$$

In view of this identity, we can easily show that for any positive integer $k$,

$$
\begin{aligned}
& Q^{2 k}=\left(\begin{array}{cc}
(X Y)^{k} & 0 \\
0 & (Y X)^{k}
\end{array}\right), \\
& \mathcal{P}_{Y X}\left(Q^{2}\right)=\sum_{k=0}^{n} a_{k} Q^{2 k}=\left(\begin{array}{cc}
\sum_{k=0}^{n} a_{k}(X Y)^{k} & 0 \\
0 & \sum_{k=0}^{n} a_{k}(Y X)^{k}
\end{array}\right) \\
& =\left(\begin{array}{cc}
\sum_{k=0}^{n} a_{k}(X Y)^{k} & 0 \\
0 & 0
\end{array}\right), \\
& \mathcal{P}_{Y X}\left(Q^{2}\right) Q=\left(\begin{array}{cc}
0 & \sum_{k=0}^{n} a_{k}(X Y)^{k} X \\
0 & 0
\end{array}\right)=\left(\begin{array}{ll}
0 & X \sum_{k=0}^{n} a_{k}(Y X)^{k} \\
0 & 0
\end{array}\right)=0 .
\end{aligned}
$$


A similar calculation yields $\mathcal{P}_{X Y}\left(Q^{2}\right) Q=0$. Furthermore, using Lemma 0 one can see that if $m_{+}=m_{-}$, then $\mathcal{P}_{X Y}(x)=\mathcal{P}_{Y X}(x)$. In view of this identity and Eq. (A.8), we have (for the case $\left.m_{+}=m_{-}\right) \mathcal{P}_{X Y}\left(Q^{2}\right)=\mathcal{P}_{Y X}\left(Q^{2}\right)=0$.

Corollary 1. Consider the $n \times n$ matrix $M$ whose elements are given by

$$
M_{i j}:=a_{j} \delta_{i, j+1}+a_{i}^{*} \delta_{i+1, j}, \quad i, j \in\{1,2, \ldots, n\}
$$

i.e.,

$$
M:=\left(\begin{array}{cccccc}
0 & a_{1}^{*} & 0 & \cdots & 0 & 0 \\
a_{1} & 0 & a_{2}^{*} & \cdots & 0 & 0 \\
0 & a_{2} & 0 & \cdots & 0 & 0 \\
\vdots & \vdots & \vdots & \ddots & \vdots & \vdots \\
0 & 0 & 0 & \cdots & 0 & a_{n-1}^{*} \\
0 & 0 & 0 & \cdots & a_{n-1} & 0
\end{array}\right) .
$$

Then the characteristic polynomial of $M$ is given by

$$
\mathcal{P}_{M}(x)= \begin{cases}\mathcal{P}_{A A^{\dagger}}\left(x^{2}\right) & \text { for } n=2 p, \\ \mathcal{P}_{A A^{\dagger}}\left(x^{2}\right) x & \text { for } n=2 p+1,\end{cases}
$$

where $A$ is the matrix with entries

$$
A_{i j}=a_{2 i-1} \delta_{i j}+a_{2 i}^{*} \delta_{i+1, j}
$$

It is a $p \times p$ matrix for $n=2 p$ and $a p \times(p+1)$ matrix for $n=2 p+1$.

Proof. Consider the following unitary transformation

$$
M \rightarrow \widetilde{M}=U^{\dagger} M U
$$

where $U$ is defined by

$$
U_{i j}= \begin{cases}\delta_{i, 2 j} & \text { for } j \leqslant p, \\ \delta_{i, 2 j-2 p-1} & \text { for } j>p .\end{cases}
$$

Substituting this equation in (A.14), we find

$$
\widetilde{M}=\left(\begin{array}{cc}
0 & A \\
A^{\dagger} & 0
\end{array}\right) .
$$

Now applying Lemma 1, we obtain Eq. (A.12). Furthermore, the coefficients of the characteristic polynomial of $M$ are functions of $\left|a_{i}\right|^{2}$ 's only.

Corollary 2. Consider the $n \times n$ complex matrix 


$$
Q:=\left(\begin{array}{cccccc}
0 & a_{1}^{*} & 0 & \cdots & 0 & a_{n} \\
a_{1} & 0 & a_{2}^{*} & \cdots & 0 & 0 \\
0 & a_{2} & 0 & \cdots & 0 & 0 \\
\vdots & \vdots & \vdots & \ddots & \vdots & \vdots \\
0 & 0 & 0 & \cdots & 0 & a_{n-1}^{*} \\
a_{n}^{*} & 0 & 0 & \cdots & a_{n-1} & 0
\end{array}\right) .
$$

Then the characteristic polynomial of $Q$ is given by

$$
\mathcal{P}_{Q}(x)=-\left(\prod_{k=1}^{n} a_{k}+\prod_{k=1}^{n} a_{k}^{*}\right)+x^{n}+\beta_{n-2} x^{n-2}+\cdots+\beta_{n-2 k} x^{n-2 k}+\cdots,
$$

where $\beta_{n-2 k}$ 's are functions of $\left|a_{i}\right|^{2}$ 's.

Proof. Using a straightforward application of the properties of the determinant, one can show that

$$
\mathcal{P}_{Q}(x)=-\left(\prod_{k=1}^{n} a_{k}+\prod_{k=1}^{n} a_{k}^{*}\right)+\mathcal{P}_{M}(x)-\left|a_{n}\right|^{2} \mathcal{P}_{M^{\prime}}(x),
$$

where $M^{\prime}$ is obtained from $M$ by removing the first and last rows and columns. Now, since $\mathcal{P}_{M}(x)$ is an odd (even) polynomial for odd (even) $n, \mathcal{P}_{Q}(x)$ will have the form given by Eq. (A.18).

Lemma 2. Let $\left(m_{1}, m_{2}, \ldots, m_{n}\right)$ be an $n$-tuple of positive integers satisfying $m_{1} \leqslant m_{2} \leqslant$ $\ldots \leqslant m_{n}, m:=\sum_{\ell=1}^{n} m_{\ell}, \delta$ is the number of times $m_{1}$ appears in $\left(m_{1}, m_{2}, \ldots, m_{n}\right), Q$ is an $m \times m$ matrix of the form (75), and $\mathcal{P}(x)$ is the characteristic polynomial of the $m_{1} \times m_{1}$ matrix $A_{n} A_{n-1} \cdots A_{2} A_{1}$. Then $Q$ satisfies

$$
\mathcal{P}\left(Q^{n}\right) Q^{n-\delta}=0 .
$$

Sketch of Proof. The proof of this lemma is very similar to the proof of Lemma 1. The idea is to multiply the block diagonal matrix $\mathcal{P}\left(Q^{n}\right)$ with a power of $Q$ so that one obtains a matrix whose entries have a factor $\mathcal{P}\left(A_{n} A_{n-1} \cdots A_{1}\right)$. Then one uses the Cayley-Hamilton theorem to conclude that the resulting matrix must vanish. One can show by inspection that the smallest nonnegative integer $r$ for which a factor of $\mathcal{P}\left(A_{n} A_{n-1} \cdots A_{1}\right)$ occurs in all the entries of $\mathcal{P}\left(Q^{n}\right) Q^{r}$ is $n-\delta$.

\section{References}

[1] E. Witten, Nucl. Phys. B 202 (1982) 253.

[2] L.E. Gendenshtein, I.V. Krive, Sov. Phys. Usp. 28 (1985) 645-666.

[3] F. Cooper, A. Khare, U. Sukhatme, Phys. Rep. 251 (1995) 267-385.

[4] G. Junker, Supersymmetric Methods in Quantum and Statistical Physics, Springer-Verlag, Berlin, 1996. 
[5] E. Witten, J. Diff. Geom. 17 (1982) 661.

[6] L. Alvarez-Gaumé, Commun. Math. Phys. 90 (1983) 161;

L. Alvarez-Gaumé, J. Phys. A: Math. Gen. 16 (1983) 4177;

P. Windey, Acta. Phys. Pol. B 15 (1984) 453;

A. Mostafazadeh, J. Math. Phys. 35 (1994) 1095.

[7] V.A. Rubakov, V.P. Spiridonov, Mod. Phys. Lett. A 3 (1988) 1337.

[8] J. Beckers, N. Debergh, Nucl. Phys. B 340 (1990) 767.

[9] D. Bonatsos, C. Daskaloyannis, Phys. Lett. B 307 (1993) 100;

N. Debergh, J. Phys. A: Math. Gen. 26 (1993) 7219;

K.N. Ilinski, V.M. Uzdin, Mod. Phys. Lett. A 8 (1993) 2657.

[10] C. Ahn, D. Bernard, A. Leclair, Nucl. Phys. B 346 (1990) 409;

L. Baulieu, E.G. Floratos, Phys. Lett. B 258 (1991) 171;

R. Kerner, J. Math. Phys. 33 (1992) 403;

S. Durand, Mod. Phys. Lett. A 8 (1993) 1795;

S. Durand, Mod. Phys. Lett. A 8 (1993) 2323;

A.T. Filippov, A.P. Isaev, R.D. Kurdikov, Mod. Phys. Lett. A 7 (1993) 2129;

N. Mohammedi, Mod. Phys. Lett. A 10 (1995) 1287;

N. Fleury, M. Rausch de Traubenberg, Mod. Phys. Lett. A 11 (1996) 2899;

J.A. de Azcárraga, A.J. Macfarlane, J. Math. Phys. 37 (1996) 1115;

R.S. Dunne, A.J. Macfarlane, J.A. de Azcárraga, J.C. Pérez Bueno, Int. J. Mod. Phys. A 12 (1997) 3275.

[11] S. Durand, Phys. Lett. B 312 (1993) 115.

[12] N.V. Borisov, K.N. Ilinski, V.M. Uzdin, Phys. Lett. A 169 (1992) 422;

A.D. Dolgallo, K.N. Ilinski, Ann. Phys. 236 (1994) 219.

[13] A. Mostafazadeh, Int. J. Mod. Phys. A 12 (1997) 2725.

[14] A. Mostafazadeh, K. Aghababaei Samani, Mod. Phys. Lett. A 15 (2000) 175.

[15] A. Mostafazadeh, Int. J. Mod. Phys. A 11 (1996) 1057.

[16] S. Durand, L. Vinet, Mod. Phys. Lett. A 6 (1991) 3165. 